\title{
Increase of Effective String Tension and Production of Strange Particles
}

\author{
Tai $\mathrm{An}^{2}$ and Sa Ben-Hao ${ }^{1,3,4}$ \\ 1. CCAST (World Lab.), P. O. Box 8730 Beijing, 100080 \\ China. \\ 2. Institute of High Energy Physics, Academia Sinica, \\ P. O. Box 918, Beijing, 100039 China." \\ 3. China Institute of Atomic Energy, P. O. Box 275 (18), \\ Beijing, 102413 China. \\ 4. Institute of Theoretical Physics, Academia Sinica, \\ Beijing 100080 China.
}

\begin{abstract}
The increase of effective string tension as a result of the hard gluon kinks on a string is investigated using a parametrization form. In this form the effective string tension increasing with energies in hadron-hadron collisions is due to the mini-jet (gluon) production in the collisions. The data of the energy dependence of the strange quark suppression factor in hh collisions are very well reproduced with this mechanism. Meanwhile, the experimental phenomena of approximate energy independence of the strange quark suppression factor in $\mathrm{e}^{+} \mathrm{e}^{-}$-annihilations are discussed.

PACS numbers: 24.10.Lx, 25.75.Dw

Keywords: Effective string tension, strange particle, Monte-Carlo generator.
\end{abstract}

\footnotetext{
${ }^{1}$ mailing address.

Email: taian@hptc1.ihep.ac.cn
} 
Originally, in the LUND fragmentation scheme [1], the strange quark suppression factor ( $\lambda$ hereafter), i.e. the suppression of $\mathrm{s}$ quark pair production in the color field with respect to $\mathrm{u}$ or $\mathrm{d}$ pair production, was assumed to be a 'constant'. This assumption was confirmed by $\mathrm{e}^{+} \mathrm{e}^{-}$experiments up to $\mathrm{Z}^{0}$ energy, except at low energies. [2] [3].

However, it has been known for years that in hadron-hadron collisions $\lambda$ is not a constant but increasing from a value of 0.2 at the ISR energies to about 0.3 at the top of the SPS energies [2]. Recent data of the p $\overline{\mathrm{p}}$ collision at $\sqrt{s}=630 \mathrm{GeV}$ from UA1 again confirmed this fact [四]. The $\lambda$ value extracted by UA1 is about 0.29 . The energy dependence of $\lambda$ indicates that there is a mechanism in hh collisions which leads to enhanced production of strange quark pairs at higher energies. So far, no quantitative description exists for the issue of the energy dependence behaviours of $\lambda$ shown in the hh collisions and $e^{+} e^{-}$-annihilations, due to our knowledge.

In this letter we propose a scenario to link the increase of the strange quark suppression factor to the effective string tension in the hh collisions based on the Lund string fragmentation model (more specifically, FRITIOF model [5] or its extension LUCIAE model [6]). The idea of this scenario is that the production of mini-jets (gluons) in a hh collision will increase the effective string tension, therefore enhance the production of s quark pairs in the string fragmentation. A parametrization form is proposed based on such a scenario, which gives a reasonable energy dependence of the strange quark suppression factor in hh collisions. This scenario thus provides a dynamic explanation for the reduction of strange quark suppression at higher energies shown in the experimental data of hh collisions.

The string tension is defined as the energy per unit length of a string. However, the existence of gluons on a string (regarded as the transverse excitation or 'kink' on a string in the Lund string fragmentation model) would wrinkle the string and give a fractal structure. Such a wrinkled string has obviously larger energy density in comparison with a string without gluon, thereby an enhanced string tension effectively [7].

The increase of the effective string tension with the appearance of gluons on a string might also be understood in two following ways: First, the string tension $\kappa$ is linked with the Regge slope $\alpha_{R}^{\prime}$ by $\kappa \sim 1 / \alpha_{R}^{\prime}$. When the pomeron exchange, which is generally looked upon as multigluon exchange, becomes important at higher energies the experiments extracted a smaller slope for the pomeron process i.e. $\alpha_{P}^{\prime}<\alpha_{R}^{\prime}$. That means the pomeron process corresponds to the 
exchange of a string with larger string tension. Second, if we are allowed to compare QCD with an abelian theory the argument that the mean energy density in an abelian theory is only a function of "the charge" in the field would yield $\kappa \sim\left(Q_{q}^{2}+2 Q_{q} Q_{g}\right) / A$, where $Q_{q}$ and $Q_{g}$ are the charges of quark field and gluon field, respectively and $A$ is the transverse size of a string. Above expression indicates again that the existence of the gluon field would enhance the string tension.

Since the question that we are concerned with is in the nonperturbative regime we can not establish a relation between the effective string tension and the gluons on a string from the first principle. Instead we will include this effect in a parametrization form.

In the Lund string fragmentation model the mean multiplicity from the fragmentation of a $q \bar{q}$ string state with mass $\sqrt{s}$ is given by

$$
\bar{n} \propto \ln \left(\frac{s}{s_{0}}\right),
$$

where the parameter $\sqrt{s_{0}}$ is of the order of a typical hadron mass. However for a multigluon string state with (n-2) gluons, indexed in a colour connected way from the $q$ (index 1 ) to the $\bar{q}$ (index $\mathrm{n}$ ), the corresponding mean multiplicity is

$$
\bar{n} \propto \ln \left(\frac{s}{s_{0}}\right)+\sum_{j=2}^{n-1} \ln \left(\frac{k_{\perp j}^{2}}{s_{0}}\right),
$$

where $k_{\perp j}, \mathrm{j}=1, \ldots(\mathrm{n}-1)$, are the transverse momenta of the emitted gluons with $k_{\perp j}^{2} \geq s_{0}$. The second term of Eq.(2) attributes to the contribution of gluons. Since the fractal structure of a string is governed by the hardest gluon on the string we then construct a quantity

$$
\xi=\frac{\ln \left(\frac{k_{\perp \max }^{2}}{s_{0}}\right)}{\ln \left(\frac{s}{s_{0}}\right)+\sum_{j=2}^{n-1} \ln \left(\frac{k_{\perp j}^{2}}{s_{0}}\right)}
$$

to represent the scale that the multigluon string is deviated from a pure $q \bar{q}$ string. We apply further the following expression to parametrize the effective string tension of the multigluon string

$$
\kappa_{e f f}=\kappa_{0}(1-\xi)^{-\alpha},
$$

where $\kappa_{0}$, the string tension of the pure $q \bar{q}$ string, is a constant and $\alpha$ is a parameter to be determined in comparison with data. 
In the Lund string fragmentation model, the $q \bar{q}$ pairs with the quark mass $m$ and the transverse momentum $p_{t}$ are produced from the colour field by a quantum tunneling process with probability

$$
\exp \left(\frac{-\pi m^{2}}{\kappa_{e f f}}\right) \exp \left(\frac{-\pi p_{t}^{2}}{\kappa_{e f f}}\right) .
$$

The above equation shows that the probability of the $s \bar{s}$ pair production with respect to a $u \bar{u}$ (or $d \bar{d}$ ) pair as well as the probability of a high $p_{t} q \bar{q}$ pair production will be enhanced in a field with larger $\kappa_{\text {eff }}$.

Assume that the width of the Gaussian transverse momentum distribution of $q \bar{q}$ pairs in the string fragmentation and the strange quark suppression factor of a string with effective string tension $\kappa_{e f f 1}$ are $\sigma_{1}$ and $\lambda_{1}$, respectively, then those quantities of a string with effective string tension $\kappa_{e f f 2}$ can be calculated from Eq.(5), i.e.

$$
\begin{aligned}
\sigma_{2} & =\sigma_{1}\left(\frac{\kappa_{e f f 2}}{\kappa_{e f f 1}}\right)^{1 / 2} \\
\lambda_{2} & =\lambda_{1}^{\frac{\kappa_{e f f 1}}{\kappa_{\text {eff } 2}}} .
\end{aligned}
$$

We see here that $\sigma$ and $\lambda$ of above two string states are related by the ratio of the effective string tensions of those two string states only. It should be noted that the discussion above is also valid for the production of the diquark pairs from the string field, i.e. the production of the diquark pairs with respect to the $q \bar{q}$ pairs will be enhanced from a string with larger $\kappa_{\text {eff }}$, therefore, more baryons (or antibaryons) will be formed in the final state.

In FRITIOF a hh collision is pictured as the multi-scattering among the partons inside the two colliding hadrons. During the collision two hadrons are excited due to longitudinal momentum transfers and/or a QCD parton-parton scattering (Rutherford Parton Scattering, RPS) if four-momentum transfer is large. The highly excited states will emit bremsstrahlung gluons according to the colourdipole formalism until the $k_{\perp}$ of the emitted gluon is smaller than a given cut-off $(\sim 1 \mathrm{GeV} / \mathrm{c})$. Thus a multigluon string state is formed and it is afterwards allowed to decay into final state hadrons according to the Lund fragmentation scheme implemented in JETSET generator [8]. LUCIAE [6] is developed based on FRITIOF by adding in more physical mechanisms like the rescattering of produced particles, the one in this paper, etc.. In $e^{+} e^{-}$annihilation a multigluon 
string state is built up by the standard QCD parton-shower scheme, which is also implemented in JETSET.

In JETSET routine which runs together with LUCIAE event generator there are model parameters PARJ(2) (the same as $\lambda$ ) and $\operatorname{PARJ}(3)$. PARJ(3) is the extra suppression of strange diquark production compared to the normal suppression of strange quark pair. Both PARJ(2) and PARJ(3) are responsible for the s quark (diquark) suppression and related to the effective string tension. Besides $\lambda$ and PARJ(3) there is PARJ(1), which stands for the suppression of diquark-antidiquark pair production in the color field in comparison with the quark-antiquark pair production and is related to the effective string tension as well. Another parameter PARJ(21) (the same as $\sigma$ ), which is the width of the Gaussian transverse momentum distribution of $q \bar{q}$ pairs in the string fragmentation, varies with $\kappa_{\text {eff }}$ too, but it is not related to the strange particle production directly.

It has been shown in the literature [9][10] [11] that the string fragmentation by JETSET with default values of PARJ(1)=0.1, PARJ(2) $=0.3$ and PARJ $(3)=0.4$ overestimates the yield of strange particles in the pp collision at $200 \mathrm{GeV} / \mathrm{c}$. Thus in this letter we first retune these parameters by comparing with the pp data of strange particle production [12]. A new set of parameters PARJ $(1)=0.046$, $\operatorname{PARJ}(2)=0.2$ and $\operatorname{PARJ}(3)=0.3$ are found for pp at $200 \mathrm{GeV} / \mathrm{c}$. We also give a new value of 0.32 for $\operatorname{PARJ}(21)$ (the corresponding default value is 0.37 ). In addition, in order to provide a value of $\lambda \simeq 0.3$ required by the $\mathrm{p} \overline{\mathrm{p}}$ data at the SPS energies [2], the parameter $\alpha$ in Eq.(4) and the $\sqrt{s_{0}}$ in Eq.(3) are determined to be about 3.5 and $0.8 \mathrm{GeV}$, respectively. Once we have determined the JETSET parameter values at $200 \mathrm{GeV} / \mathrm{c}$ we can then apply Eq. (4) and Eq.(6) to calculate the values of these parameters for other energies.

Taking the changing behavior of those JETSET parameters, mentioned above, into account we have compared the calculated results of particle production with the data in Tab.1 and Tab.2 for the pp collision at $200 \mathrm{GeV} / \mathrm{c}$ [12 and $\mathrm{p} \overline{\mathrm{p}}$ at $\sqrt{s}=540 \mathrm{GeV}$ [13], respectively. The agreement between the data and the results of LUCIAE is good. The four JETSET parameters for $\mathrm{p} \overline{\mathrm{p}}$ at $\sqrt{s}=540 \mathrm{GeV}$ are found to be $\operatorname{PARJ}(1)=0.115, \operatorname{PARJ}(2)=0.304, \operatorname{PARJ}(3)=0.406$, $\operatorname{PARJ}(21)=0.376$ (the corresponding JETSET parameters for the pp collision at $200 \mathrm{GeV} / \mathrm{c}$ have already been determined in the last paragraph). The HIJING results [10] are also included in Tab.1 and Tab.2 for comparison. HIJING used JETSET with default param- 
eters (i.e. $\lambda=0.3$ etc.) to fragment a string, so it overestimates the production of strange particles in pp at $200 \mathrm{GeV} / \mathrm{c}$, but reproduces the multiplicities of strange particles in $\mathrm{p} \overline{\mathrm{p}}$ at $\sqrt{s}=540 \mathrm{GeV}$.

The energy dependence of $\lambda$ in hh collisions is also calculated and the results are shown in Fig.1 together with the experimental data from 21. One sees from this figure that the agreement between data and the results of LUCIAE is reasonably good.

In hh collisions there are two strings formed before fragmentation. The $\lambda$ value calculated above is the mean value of the two strings. It is needed to point out that the increase of the effective string tension with increasing of energies in hh collisions is due to the production of high $k_{\perp}$-gluons from either the RPS or the bremsstrahlung radiation of the colour dipoles (both of which have been included in FRITIOF and/or LUCIAE).

In Fig.2 we give the calculated results (solid line) of the energy dependence of $\lambda$ in $e^{+} e^{-}$-annihilations and the corresponding data from [2] (black circle) and [3] (open circle). One sees from this figure that the constant $\lambda$ value around 0.3 only occurs in the energy region of $\sqrt{s}>30 \mathrm{GeV}$ and the agreement between data and calculated results is good. However, due to the large error bars of the data points in the low energy region it is hard to make any conclusion for the energy dependence of $\lambda$ in this region. But the threshold effect is seen obviously here below $\sqrt{s}<30 \mathrm{GeV}$, while in hh collisions the saturation comes much later at $\sqrt{s} \simeq 200 \mathrm{GeV}$.

When Lund model was developed to study hh collisions it was assumed that a string formed in hh collisions (quark-diquark string) is the same type of the string as formed in $e^{+} e^{-}$- annihilations (quarkantiquark string) - the jet universality. But detailed analysis reveals certain differences of this two types of string states due to the different physical processes involved in the formation of the strings. First, contrary to an $e^{+} e^{-}$-annihilation the energy and momentum in a hh collision are not localised in a pointlike color charge but spread over some extended region. Any bremsstrahlung emission ¿from an extended source will necessarily be influenced by this extension ('the form factor'). Second, the hard RPS gluon jets produced in a hh collision will also greatly affect the topologic structure of a string formed. Third, only about half of the incoming energy can be used in the particle production in hadron-hadron collisions and the rest is carried away by the leading particles, unlike in an $e^{+} e^{-}$annihilation where all the incoming energy can be used to produce secondary hadrons. Those differences might explain the difference 
of the energy dependence behaviours of $\lambda$ in $e^{+} e^{-}$-annihilations and hh collisions.

It is worth pointing out that, since Eq.(14) can be approximated by $\kappa_{e f f} \approx \kappa_{0}(1+\alpha \xi)$ thus in comparison with the formula from the abelian theory $\xi$ might be related to the ratio of charges of gluon and quark field $Q_{g} / Q_{q}$.

In summary, we have proposed a scenario inspired by the Lund string fragmentation model to investigate the reduction of strange quark suppression shown in experimental data of strange particle production in hh collisions. We obtain an appropriate parametrization form to characterize the relationship between the effective string tension and the hard gluons on a string. This scenario reproduces reasonably the energy dependence of the strange quark suppression factor in hh collisions and the nearly energy independence (above $\sqrt{s}>30 \mathrm{GeV})$ in $e^{+} e^{-}$- annihilations.

\section{Acknowledgment}

This work is partly supported by the national Natural Science Foundation of China. 


\section{References}

[1] B. Andersson, G. Gustafson, G. Ingelman and T. Sjöstrand, Phys. Rep. 97, 31 (1983).

[2] A. K. Wróblewski, Proceedings of the 25th International conference on HEP, p. 125, Singapore, 1990.

[3] G. Alexander et.al., OPAL Colla., Phys. Lett. B264, 467 (1991).

[4] G. Bocquet etal., UA1 Colla., Phys. Lett., B366, 447 (1996).

[5] H. Pi, Comp. Phys. Commu. 71, 173 (1992).

[6] Sa Ben-Hao and Tai An, Comp. Phys. Commu., 90, 121 (1995).

[7] T. Sjöstrand's lecture note in Lund.

[8] T. Söstrand, Comp. Phys. Commu. 39, 374(1986).

[9] B. Andersson, An Tai and Ben-Hao Sa, Z. Phys., C70, 499 (1996);

Sa Ben-Hao and Tai An, Phys. ReV. C55, 2010 (1997).

[10] V. Topor Pop, M. Gyulassy, X. N. Wang, A. Andrighetto, M. Morando, F. Pellegrini, R. A. Ricci and G. Segato, Phys. ReV., C52,1618 (1995).

[11] M. Gaździcki, and U. Heinz, Phys. Rev., C54, 1496 (1996).

[12] M. Gazdzicki and O. Hansen, Nucl. Phys. A528, 754 (1991).

[13] G. J. Alner et al., Phys. Rep. 154, 247 (1987); D. R. Ward, in "Proton Antiproton Collisions", Advanced Series on Directions in High Energy Physics, Vol. 4, edited by G. Altarelli and L. Di Lella (World Scientific, Singapore, 1989), p85. 


\section{Figure Captions}

Fig. 1 The energy dependence of $\lambda$ in hh collisions. The data are taken from [2].

Fig. 2 The energy dependence of $\lambda$ in $e^{+} e^{-}$-annihilations. The data points are taken from [2] (black circle) and [3] (open circle), respectively.

Table 1. Particle multiplicities (full phase space) for the pp interaction at $200 \mathrm{GeV} / \mathrm{c}$ are compared with experimental data 112 and HIJING calculation.

\begin{tabular}{cccc}
\hline \hline particle type & Expt. data & LUCIAE & HIJING \\
\hline$\pi^{-}$ & $2.62 \pm 0.06$ & 2.79 & 2.65 \\
$\pi^{+}$ & $3.22 \pm 0.12$ & 3.24 & 3.23 \\
$\pi^{0}$ & $3.34 \pm 0.24$ & 3.61 & 3.27 \\
$h^{-}$ & $2.86 \pm 0.05$ & 3.01 & 3.03 \\
$K^{+}$ & $0.28 \pm 0.06$ & 0.23 & 0.32 \\
$K^{-}$ & $0.18 \pm 0.05$ & 0.16 & 0.25 \\
$\Lambda+\Sigma^{0}$ & $0.096 \pm 0.015$ & 0.111 & 0.165 \\
$\bar{\Lambda}+\bar{\Sigma}^{0}$ & $0.013 \pm 0.005$ & 0.015 & 0.037 \\
$K_{s}^{0}$ & $0.17 \pm 0.01$ & 0.18 & 0.27 \\
$p$ & $1.34 \pm 0.15$ & 1.00 & 1.45 \\
$\bar{p}$ & $0.05 \pm 0.02$ & 0.04 & 0.12 \\
\hline \hline
\end{tabular}


Table 2. Particle multiplicities (full phase space) for the $\mathrm{p} \overline{\mathrm{p}}$ interaction at $\sqrt{s}=540 \mathrm{GeV}$ are compared with experimental data 13] and HIJING calculation.

\begin{tabular}{cccc}
\hline \hline particle type & Expt. data & LUCIAE & HIJING \\
\hline All charged & $29.4 \pm 0.3$ & 28.4 & 28.2 \\
$K^{0}+\bar{K}^{0}$ & $2.24 \pm 0.16$ & 2.62 & 1.98 \\
$K^{+}+K^{-}$ & $2.24 \pm 0.16$ & 2.72 & 2.06 \\
$p+\bar{p}$ & $1.45 \pm 0.15$ & 2.62 & 1.55 \\
$\Lambda+\bar{\Lambda}$ & $0.53 \pm 0.11$ & 0.58 & 0.50 \\
$\Sigma^{+}+\Sigma^{-}+\bar{\Sigma}^{+}+\bar{\Sigma}^{-}$ & $0.27 \pm 0.06$ & 0.32 & 0.23 \\
$\Xi^{-}$ & $0.04 \pm 0.01$ & 0.032 & 0.037 \\
$\pi^{+}+\pi^{-}$ & $23.9 \pm 0.4$ & 22.69 & 23.29 \\
$<K_{s}^{0}>$ & $1.1 \pm 0.1$ & 1.32 & 0.99 \\
$\pi^{0}$ & $11.0 \pm 0.4$ & 12.82 & 13.36 \\
\hline \hline
\end{tabular}




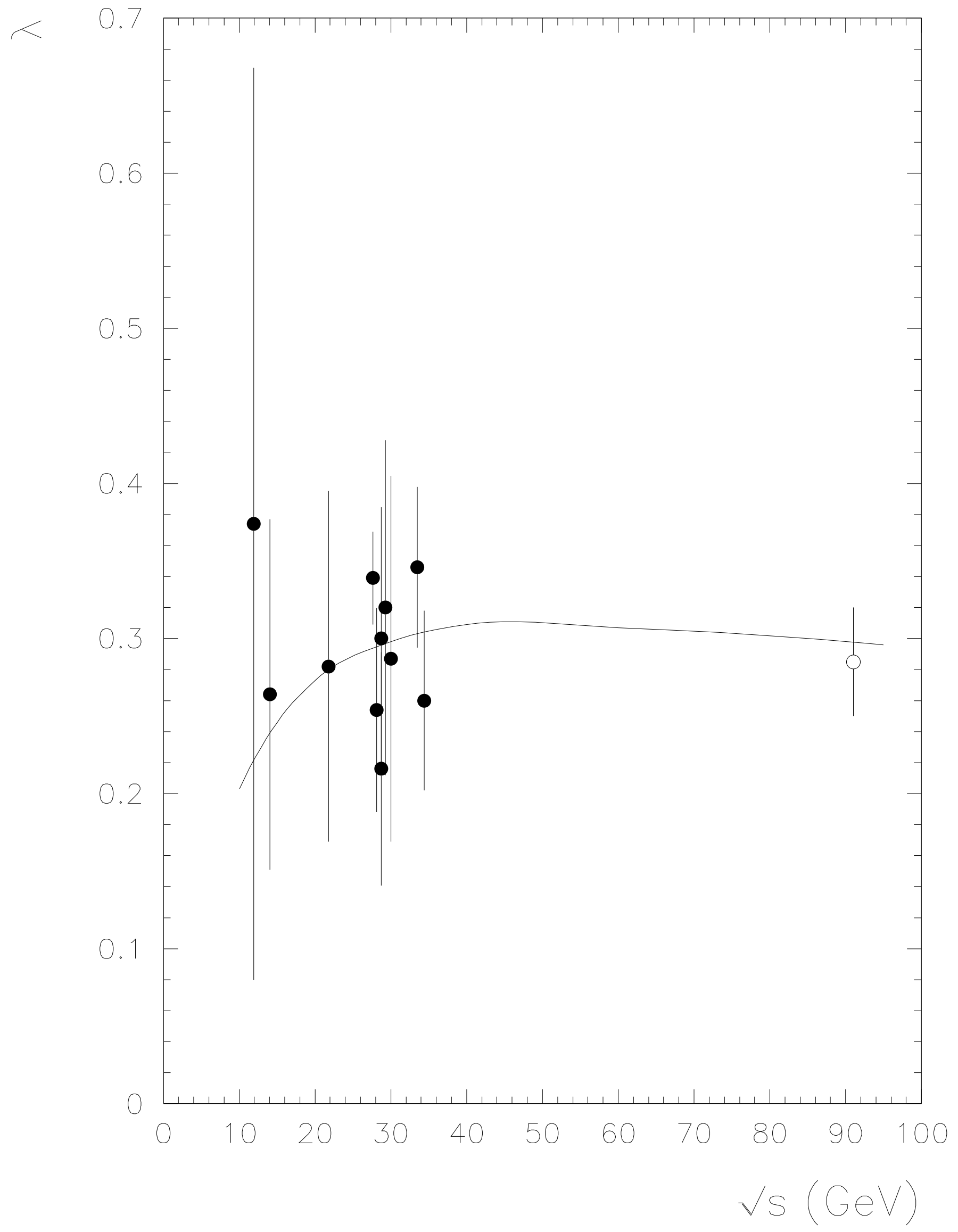




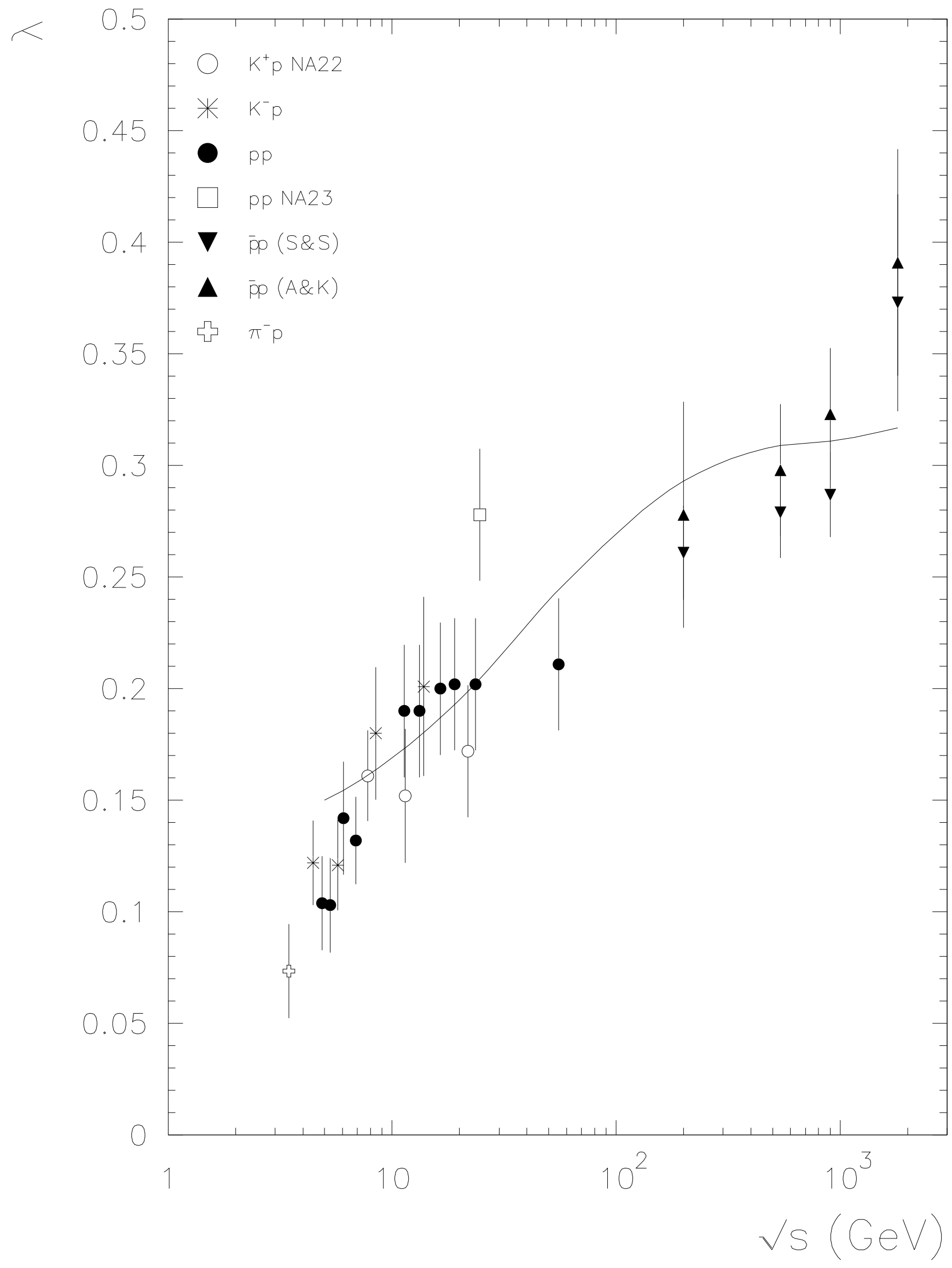

